\title{
Quelques problèmes non résolus de la théorie des fonctions caractéristiques.
}

par Paul Lẃvy (Paris, France)

A M. Enrico Bompiani pour son Jubilé scientifique.

Résumé. - Le plus important des problèmes traités est celui du prolongement d' une fonction caractéristique donnée dans un intervalle ou dans une réunion d'intervalles. Malgré un beau théorème de $\mathrm{M}$. KREIN, ce problème ne peut pas être consdéré comme résolu. On donne ici quelques conditions qui sont, soit nécessaires, soit suffisantes pour la possibitité et pour l'unicité du prolongement.

I. Nous désignerons par $C$ la classe des fonctions $\varphi(z)$ de la variable réelle $z$ définies par la formule

$$
\varphi(z)=\int_{-\infty}^{+\infty} e^{i x z} d E(z)
$$

où $F(z)$ est une fonotion non décroissante, bornée, et non constante, de sorte que l'on a

$$
c=\varphi(0)=\int_{-\infty}^{+\infty} d F(x)>0 .
$$

A ce facteur c (qui ne jouera pas dans la suite un rôle essentiel) près, et à cela près qu' en calcul des probabilités on suppose généralement $F(-\infty)=0$, $F(x)$ et $\varphi(z)$ sont respectivement la fonction de répartition et la fonction caractéristique da calcul des probabilités.

On sait reconnattre si une fonction $\varphi(z)$ donnée appartient à la classe $C$. Il faut d'abord $q u^{\prime}$ elle soit continue, que $\varphi(z)$ et $\varphi(-z)$ soient imaginaires conjugués, de sorte que l'intégrale

$$
I(x, Z)=\frac{1}{2 \pi i} \int_{-Z}^{Z}\left(1-e^{-i x z}\right) \varphi(z) \frac{d z}{z}
$$


qui, dans le cas où $\varphi(z)$ est réel (done pair), se réduit à

$$
\frac{1}{2 \pi} \int_{-Z}^{Z} \frac{\sin x z}{z} \varphi(z) d z,
$$

représente un nombre réel. Il est alors nécessaire et suffisant. pour que $\varphi(\cdot) \in C, q$ u'elle tende pour $Z$ infini vers une limite, qui alors représente $F(x)-F(0)\left({ }^{2}\right)$, et que cette limite soit nne fonetion non décroissante de $x$.

On sait l'importance qu'a en calcul des probabilités la formulo

$$
H(x)=\int_{-\infty}^{+\infty} G(x-y) d F(y)
$$

qui définit la fonction de répartition de la somme de deux varíables aléatoires indépendantes dont les fonctions de répartition sont $F(x)$ et $G(y)$.

On sait aussi que, si on fait intervenir les transformeses de FourIerStieltjes $\varphi(z), q(z)$ et $p(z)$ de $F(x), G(x), H(x)$, cette relation prend la forme simple

$$
p(z)=q(z) \varphi(z) .
$$

Il en résulte que, si les fonotions $q(z)$ et $\varphi(z)$ appartiennent à la classe $C$, il en est de même du produit $p(z)$.

Le problème qui est l'objet de ce travail est la résolution de l'équation (4) par rapport a $F(x)$. On a pu le croire résolu, puisqu'il équivaut, du moins si $G(-\infty)=H(-\infty)=0\left({ }^{2}\right)$, à la recherche d' une fonction $\varphi(z)$ qui vérifie l'équation (5), et qui de plus appartienne à la classe $C$, si on veut que la fonction $F(x)$ soit non décroissante. Or comme nous l'avons rappelé plus haut, on sait reconnaître si une fonction donnée appartient à la classe $C$. Le problème posé est ainsi résolu dans le cas où l'ensemble $\mathcal{E}$ où $q(z) \neq 0$ comprend tout l'axe des $z$; la fonction $\varphi(z)$ est en effet connue dans ce cas sur tout cet axe. Sa solution est aussi évidente si $\mathfrak{G}$ est partout dense; la fonotion cherchée doit en effet être continue, de sorte que, s'il y a une solution, étant

(1) Pour les points de discontinuité de $F(x)$, cela n'est vrai que si l'on a défini $F^{\prime}(x)$ par la formule

$$
F(x)=\frac{1}{2}[F(x-0)+F(x+0)]
$$

(2) Sans cette condition, (4) équivant à (5), complété par la condition $H(-\omega)=c G(-\omega)$, $q u i$, puisque $c=\frac{p(0)}{q(0)}$, est une condition imposée aux données. 
connue sur l' ensemble $\mathcal{E}$ elle l'est aussi sur snr sa fermeture $\overline{\mathcal{G}}$, donc, dans le cas considéré, sur tout l'axe des z. Mais, si $\mathcal{G}$ n'est pas partout dense, il $\mathbf{s}^{\prime}$ agit de prolonger dans l'ensemble complémentaire $\overline{\mathcal{C}^{c}}$ la fonction d'abord obtenue sur $\overline{\mathcal{G}}$. Il s'agit de savoir si ce prolongement est possible; dans l'affirmative, il s'agit de savoir s'il est unique, et, suivant la réponse à cette question, de déterminer la solution ou la forme générale des solutions ( $\left.{ }^{3}\right)$.

A. Khintchine a montré en 1936, par un exemple que nous rappellerons au $\mathrm{n}^{\circ}$. 2, qu'il peut y avoir plusienrs solutions. Je n'ai attiré l'attention qu'en 1959 (4) sur le fait que cet exemple conduisait à poser un problème qui n'est pas résolu par la formule (5). Il n'est toujours pas résolu et n' est pent-c̀tre pas résoluble [voir la note (3)]. Je me propose senlement, dans ce travail, de montrer qu'il y a beacoup d'antres cas d'indétermination que

(3) Depuis que ce travail a été rédigé, M. E. Lukacs a attiré mon attention sur un heau théorème de M. Kreis, publié dès 1940 dans lus Comptes rendus de l'Académie des Sciences de l'U.R.S.S. N. s. 26, p. 17.22. Dans le mẻme volume (p. 860-865), D. Rarkov a donné une nouvelle démonstration de ce thérème, dont voici l'énoncé:

Une fonction $\varphi_{0}(z)$ étant donnée, hermitienne et continue, dans $(-A,+A)$, pour qu'il existe une fonction $\varphi(z)$ de la classe $C$ egale $a \varphi_{0}(z)$ dans cet intervalle, il fant et il suffit que, quelle que soit la fonctions $\tau(z)$ réulle, monotone et hornée dans $(0,+A)$, on ait

$$
J(A)=\int_{0}^{A} \int_{0}^{A} \varphi_{0}\left(z-z^{\prime}\right) d \tau(z) d \tau\left(z^{\prime}\right) \geq 0 .
$$

De plus la condition $J(A)=0$ [sauf si $\tau($ a $)$ est constant], jointe i une autre condition moins simple, est nécessaire et suffisante pour que le prolongement de $\varphi_{0}(z)$ soit indétérminé.

Pour $A$ infini, on retrouve le célèbre théorème de $\mathrm{S}$. Bochner. Ce n'est pas réduire l'importance de ces théorèmes que d'observer qu'ils ne font que ramener le problème posé à un autre, et cela dans le cas où $\mathscr{E}=(-A,+A)$. Ils ne le résolvent pas, et la question posce dans la texte reste entière. Rien ne prouve d'ailleurs qu'il puisse y avoir une condition explicite, analogue à celle rappelée plus haut pour $A$ infini, qui résolve pratiquement le probleme.

Le théorème de Knain suggère une généralisation, obtenue en remplaçant $(0, A)$ par n'importe quel ensemble formé $\bar{e} \subset(0, \infty)$. Si $z$ et $z^{\prime}$ décrivent indépendamment $\bar{e}, z-z^{\prime}$ décrit un ensemble fermé $E ; J(A)$ est remplacé par une intégrale $J(A, e)$, et la condition pour que $p(z)$, donné dans $\bar{E}$, soit prolongeable dans $(-\infty,+\infty)$, serait $J(A, e) \geq 0$. Cette condi. tion est manifestement nécessaire; cela résulte du théorème de Bochner appliqué aux fonc. tions $\tau(z)$ qui ne varient que dans $e$. Il nous semble probable qu'elle est aussi suffisante. Mais bien entendu on ne peut obtenir ainsi qu' un enoncé applicable si $\vec{E}$ est la somme directe d'un ensemble $\bar{e}$ et de son symétrique par rapport à l'origine. Un tel ensemble est nécessairement son propre symétrique par rapport à l'origine; mais cette symétrie est sans importance dans le problème considéré, puisque, si la fonction hermitienne $\varphi(z)$ est connue dans un ensemble quelconque $E$, elle l'est dans $\bar{E}$, dans l'ensemble $\bar{E}^{\prime}$ symétrique de $\bar{E}$ et par suite dans la réunion de $\bar{E}$ et $\bar{E}$.

(4) P. LÉvY, Esquisse d'une théorie de la multiplication des variables alétoires *Ann. Ec. norm. sup. $", 76$ (1959), p. 59-82. 
celui signalé par le savant russe, et que dans ces cas, la solution présente un très grand degré de généralité. Il est d'ailleurs évident que, s'il y a deux solutions distinctes $\varphi_{0}(z)$ et $\varphi_{1}(z)$. il y a une infinité de solutions $\lambda \varphi_{0}(z)+\left(1-\lambda_{1}\right)$ $\varphi_{1}(z)(0 \leq \lambda \leq 1)$.

Le lecteur remarquera qu' un problème analogue à celui que nous con. sidérons. mais beacoup plus simple, se pose pour la classe des fonctions don. uées par la formule (1), si $F(x)$ est une fonction à variation bornée, à cela près quelconque.

II. Rappelons d'abord l'exemple de KHINTCHINe. Il prend pour $q(z)$ la fonction

$$
\omega(z)=\operatorname{Max}(0,1-|z|)=\frac{4}{\pi} \int_{0}^{\infty} \sin ^{2} \frac{x}{2} \cos z x \frac{d x}{x^{2}}
$$

$q$ ui est évidemment une fonction caractéristique, nulle pour $|z| \geq 1$. Si alors on prend pour $p(z)$ la fonction $\omega^{2}(z)$, l'équation (5) admet pour solution, non seulement $f_{1}(z)=\omega(z)$, mais toutes les fonctions caractéristiques égales à $\omega(z)$ dans $(-1,+1)$, et en particulier la fonction $\varphi_{2}(z)$, de période 2 , et égale à $\omega(z)$ dans $(-1,+1)$. Son développement en série de Fourier

$$
\varphi_{2}(z)=\frac{1}{2}+\frac{4}{\pi^{2}}{ }_{0}^{\infty} \frac{\cos (2 p+1) \pi z}{(2 p+1)^{2}}
$$

montre que c' est bien une fonetion caractéristique $\left({ }^{5}\right)$.

III. Nous allons, pour généraliser cet exemple, définir d'autres fonctions appartenant au sous ensemble $C_{a}$ de la classe $C$ défini par la condition que $\mathscr{E}$ se réduise à un intervalle $(-a,+a)$.

Cunsidérons d'abord les fonctions

$$
\begin{gathered}
Q(z)=\int_{0}^{\infty} \omega\left(\frac{z}{u}\right) d G(u)=\int_{|z|}^{\infty}\left(1-\frac{|z|}{u}\right) d G(u), \\
q(z)=\int_{0}^{\infty} \omega\left(\frac{z}{u}\right) \varphi(z, u) d G(u),
\end{gathered}
$$

(5) Un problème qui pent être intéressant est l'étude de la nature arithmétique des fonctions caractéristiques $\omega(z)$ et $\varphi_{2}(\varepsilon)$. Il semble bien qu'elles soient indécomposables, c'esta-dire qu'elles ne peuvent être mises sous la forme du produir de deux fonctions caracté. ristiques que si un des deux facteurs est de la forme e etz. Mais cela n' est pas démontré. 
où $G(u)$ est une fonction non décroissante bornée, et où $\varphi(z, u)$ est une fonction caractéristique dépendant d'une manière quelconque du paramètre $u$, à cela près que l'intégrale duit avoir un sens. Ce sont évidemment des fonctions de la classe $C$, et qui appartiennent à $C_{a}$ si $G(u)$ est constant dans $(a, \infty)$. Elles tendent vers zéro pour $z$ infini $\left({ }^{\circ}\right)$. Nous désignerons par $K$ l'en* semble des fonctions $q(z)$ de la forme (9), et par $K_{a}$ le sous-ensemble obtenu en supposant $G(u)$ constant dans $(a, \infty)$.

Les fonctions $Q(z)$ sont, au facteur $Q(0)$ près, les fonctions caractéristiques convexes de Pólya : ce sont des fonctions de $|z|$ bornées, non croissantes, convexes, et $\geq 0$. On sait $q u$ 'inversement, si une fonction de Póry s'annule à l'infini, elle est de la forme (8) $1^{7}$ ); il résulte de la convexité de $Q(z)$ dans $(0, \infty)$, et de ce que $Q(z)<Q(0)$ pour $z \neq 0$, que cette fonction a toujours a l'origine une dérivée à droite $Q^{\prime}(+0),<0$ et $\geq-\infty$. La courbe qui la re. présente a donc, pour $z=0$, un point anguleux ou un point de rebroussement.

D'après les formules $(8)$ et $(9), q(z)$ est majoré parQ(z). Done si $0<m$ $\checkmark\left|Q^{\prime}(+0)\right|$, il existe un voisinage de l'origine où

$$
R[q(z)] \leq|q(z)| \leq Q(0)-m|z|
$$

Cette propriété, et le fait que $q(z)$ s'annule à l'infini, montrent que la classe $K$ est définie dans $C_{1}$ par des conditions assez restrictives, qui éliminent en particulier toutes les fonctions dérivables pour $z=0$. Il ne semble d'ailleurs pas facile de trouver des conditions nécessaires et suffisantes pour $q u^{\prime}$ une fonction donnée $q(z)$ appartienne à $K$. La convexité dans $(0, \infty)$, qui caractérise les fonctions de. Pólya, n'est plus une condition nécessaire. Ainsi, si $\varphi(z)$ est une fonction caractéristique réelle, $<1$ pour $z \neq 0$, et dérivable à l'origine [done $\varphi^{\prime}(0)=0$ ] et si $q(z)=\omega(z) \varphi(z)$, on a $q^{\prime}(+0)=-1$ et, pour $0<z<1, q(z)<q(0)-z$, de sorte que l'are de courbe $y=q(z)$, $0<z<1$, est situé au dessous de sa tangente au point $z=0$. Cette propriété subsiste naturellement, dans des cas étendus, pour les fonctions de la forme (9).

Remarquons que la représentation d'une fonction $Q(z)$ par la formule (8) est unique. La fonction $G(u)$ est en effet définie, à une constante près, par la formule

$$
G(z)=\int z d Q^{\prime}(z) \quad\left[Q^{\prime}(z)=\frac{d Q(z)}{d z}\right]
$$

(c) On remarque que, pour $\lambda=0$, $\omega(\lambda z)$ se réduit a $\omega(0)=1$. Mais ce terme ne pent pas figurer dans les intigrales (8) et (9).

(i) Si une telle fonction avait à l'infini une valeur $c>0$, il faudrait ajouter le terme $\cos (0)$. 
Si elle est dérivable, sa dérivée est $G^{\prime}(z)=z Q^{\prime \prime}(z)$. Au contraire, on ne doit pas s'attendre à ce que la représentation d'une fonction $q(z) \in K$ par la formule (9) soit unique. Il se peut $q u^{\prime}$ il $y$ ait des cas d'unicité ( $\left.{ }^{8}\right)$. Mais il est évident qu'en général la seule donnée de $q(z)$ ne suffit pas à déterminer $\varphi(z, u) d G(u)$. Il est d'ailleurs facile de donner des exemples de représentations multiples.

Ainsi, en posant:

$$
\begin{aligned}
& q_{\nu}(z)=\int_{0}^{\infty} \omega\left(\frac{z}{u}\right) \varphi_{\nu}(z, u) d G_{\nu}(u) \quad(v=1,2, \ldots, n), \\
& P_{n}(z)=q_{2}(z) q_{2}(z) \ldots q_{n}(z)=q_{\nu}(z) p_{n, \nu}(z)
\end{aligned}
$$

on a une infinité de représentations de $p_{n}(z)$, obtenues en combinant les $n$ représentations, en général linéairement indépendantes:

$$
P_{n}(z)=\int_{0}^{\infty} \omega\left(\frac{z}{u}\right) \varphi_{\nu}(z, u) p_{n, v}(z) d G_{\nu}(z)
$$

Ponr un produit infini convergent, on a de même une infinité de représentations linéairement indépendantes.

Citons encore l'exemple des fonctions de Póra $e^{-|z|^{\alpha}}(0<\alpha \leq 1)$. Quel que soit $\lambda \in(0,1)$, en mettant $e^{-\lambda|z|^{\alpha}}$ sous la forme (8), on a

$$
e^{-|z|^{\alpha}}=\alpha \lambda \int_{0}^{\infty} \omega\left(\frac{z}{u}\right)\left(\alpha \lambda u^{\alpha}+1-\alpha\right) u^{\alpha-1} e^{-\lambda u^{\alpha}-(1-\lambda)|z|^{\alpha}} d u,
$$

ce qui, pour $\alpha=1$, donne

$$
e^{-|z|}=\lambda^{2} \int_{0}^{\infty} m\left(\frac{z}{u}\right) u e^{-\lambda \mu-(1-\lambda)|z|} d u .
$$

On a ainsi, pour la même fonction, des représentations linéairement indépendantes, qui dépendent du paramètre continu $\lambda$, et leurs combinaisons linéaires donnent un ensemble très vaste de représentations de la même fonction.

(8) Nous nous sommes en particulier demande si ce n'est pas le cas pour la fonction $\omega(z)$, et plus généralement pour les sommes finies de termes de la forme $C \omega(\lambda z)$. Ce problème n'est pas résolu. 
IV. Nous allons maintenant considérer les classes $\Gamma^{\prime}$ et $K^{\prime}$ de fonctions $Q(z)$ et $q(z)$ definies par les formules (8) et (9) prises au sens large, e' est-à-dire que $G(u)$, au lieu d'etre une fonction monotone, sera une fonction à variation bornée quelconque $\left({ }^{\circ}\right)$. D'après la formule $(11), Q^{\prime}(z)$ et $G(z)$ varient dans le mème sens pour $z>0$, de sorte $q \mathbf{n}^{\prime}$ on pent définir par la formule (8) des fonctions $Q(z)$ pour lesquelles le sens de variation de $Q^{\prime}(z)$ change aussi souvent qu' on le voudra et qui ne seront pas des fonctions de Pómya. Pourtant, comme nous allons le voir, elles peuvent appartenir à la classe $C$, et mème it $C_{1}$ (done aussi a n'importe quelle classe $C_{a}$ ).

D' après la formule $(6), Q(z)$ est, à un facteur constant près, la transformée de Fourier de

$$
\int_{0}^{\infty} \sin ^{2} \frac{u x}{2} \frac{d G(u)}{u x^{2}}
$$

et il s'agit de montrer que cette exprcssion peut ètre toujours $\geq 0$ sans que $G(u)$ soit monotone. L'exemple le plus simple est sans doute celui de l'expression

$$
4 \sin ^{2} \frac{x}{2}-\sin ^{2} x=4 \sin ^{4} \frac{x}{2} \geq 0
$$

qui montre que la fonction $2(2 z)-(b)$ appartient à la classe $C$, done aussi ì $C_{1}$, puisqu'elle est nulle pour $|z|>1$. On déduit de même de la formule

$$
\left|\frac{\sin n x}{\sin x}\right|=\left|\sum_{1}^{n} \cos (n+1-2 p) x\right| \leq n
$$

que, pour tout $n$ entier $>1$, on a $|\sin n x| \leq n|\sin x|$, d'où

$$
n^{2} \sin ^{2} x-\sin ^{2} n x \geq 0,
$$

et que par suite la fonction $n \omega(n z)-\omega(z)$ appartient à $C_{1}$. Ce résultat est d'aillenrs le meilleur possible, puisque, pour $c<n^{2}$, on a

$$
\frac{c \sin ^{2} x-\sin ^{2} n x}{x^{2}} \rightarrow c-n^{2}<0 \quad(x \rightarrow 0) .
$$

(') On peut même généraliser davantage, puisqu'il suffit que, par les formules $d Q^{\prime}(z)=\frac{d G(z)}{z}, d Q(z)=Q^{\prime}(z) d z$, on puisse déterminer une fonction $Q(z)$ borné à l'origine et s'annulant a l'infini. Mais sela ne nous sera pas nécessaire. 
Plus généralement, cherchons à quelles conditions la somme

$$
S(x)=\sum_{1}^{p} \frac{a_{h}}{\lambda_{h}} \sin ^{2} \lambda_{h} x-\sum_{1}^{q} \frac{b_{h}}{\mu_{k}} \sin ^{2} \mu_{k} x \quad\left(a_{h}, \lambda_{h}, b_{h}, \mu_{k}>0\right)
$$

est toujours $\geq 0$. Décomposons-la en une somme $\Sigma s_{y}(x)$ de termes périodiques, à périodes $\omega_{\nu}$ deux à deux incommensurables. Il faut que chaque $s_{\nu}(x)$ ait la pro. priétéindiquée. Si en effet $s_{y}(x)$ était $\leq c<0$ pour $x=\xi$, il le serait pour tous les $x \equiv \xi$ (mod. $\omega_{y}$ ), et on pourrait, d'après le théorème de KRoneoKER trouver un de ces $x$ pour lequel tous les $s_{\rho}(x)$ d'indices $\rho \neq v$ seraient arbitrairement petits. Alors $S(x)$ serait $<0\left({ }^{10}\right)$.

Nous pouvons donc considérer séparément chaque somme partielle $s_{\nu}(x)$, c'est-à-dire supposer tous les rapports $\frac{\lambda_{h}}{\mu_{k}}$ rationnels. Soit alors $\delta$ le plus grand commun diviseur des $\lambda_{h}$. Tous les termes positifs s'annulant si $x$ est multiple de $\frac{\pi}{\delta}$, pour que $S(x) \geq 0$, il faut que les termes négatifs s'annulent en même temps, e'est-à-dire que tous les $\mu_{k}$ soient anssi multiples de $\delta$. En d'autres termes, chaque $\mu_{k}$ doit être une combinaison linéaire à coefficients entiers des $\lambda_{h}$.

Cette condition nécessaire étant remplie par les $\lambda_{h}$ et les $\mu_{k}$ pour chaque somme partielle $s_{v}(x)$, et les $a_{h}$ étant des nombres positifs donnés, la condition nécessaire et suffisante pour que chaque $s_{v}(x)$, et par suite $S(x)$, soit toujours $\geq 0$, est que les $b_{k}$ soient assez petits. La forme exacte de cette condition est d'ailleurs assez compliquée. L'exemple d'une expression telle que $\sin ^{2} 3 x+\sin ^{2} 5 x-3 \sin ^{2} 2 x$ montre que, même pour une somme $S(x)$ réduite à une somme partielle $s_{y}(x)$, il ne suffit pas qu' elle soit $>0$ pour $x$ très petit pour l'être quel que soit $x$, comme e'était le cas pour $c \sin ^{2} x-\sin ^{2} n x$. On voit seulement que, le rapport

$$
\frac{\sin ^{2} 2 x}{\sin ^{2} 3 x+\sin ^{2} 5 x}
$$

étant borné, il a un maximum $m$, de sorte que, pour $c \leq \frac{1}{m}$, on a

$$
\sin ^{2} 3 x+\sin ^{2} 5 x-c \sin ^{2} 2 x \geq 0
$$

(10) On n' Gchappe pas à cette conclusion en introduisant des séries infinies; une série convergente $\Sigma a_{h} \sin ^{2} \lambda_{k} x$ ne peut pas majorer $\sin ^{2} x$ si tous les $\lambda_{h}$ sont irrationnele. 
Il existe ainsi des types très variés de sommes $S(x)$ toujours $\geq 0$, auxquelles correspondent des sommes

$$
\sum_{\Sigma}^{p} a_{h} \omega\left(\frac{z}{\lambda_{h}}\right)-\sum_{1}^{q} b_{k} \omega\left(\frac{z}{\mu_{h}}\right)
$$

qui appartiennent à la classe $C$, malgré la présence de coefficients négatifs. Si les $\lambda_{h}$ et les $\mu_{k}$ sont tous $\leq 1$, elles appartiennent à $C_{1}$.

Choisissons, parmi ces fonctions, une famille de fonctions $\mathbf{Q}(z, u)$ dépendant du parametre $u$, et remplaçons $\omega\left(\frac{z}{u}\right)$ par $\Omega(z, u)$ dans les expressions (8) et (9). On obtient de nouvelles fonctions

$$
\begin{aligned}
& Q(z)=\int \Omega(z, u) d H(u), \\
& q(z)=\int \Omega(z, u) \varphi(z, u) d H(u),
\end{aligned}
$$

qui, si la fonction $H(u)$ est non décroissante, appartiennent $\grave{a}$ la classe $C$, donc à la classe $C_{a}$ si tous les $\lambda_{h}$ et $\mu_{k}$ qui interviennent sont $\leq a$. La variation de $\Omega(z, u)$ avec $u$ est, comme oelle de $\varphi(z, u)$ soumise à la seule restriction que ces intégrales aient un sens. $Q(z, u)$ peut etre, comme dans la formule (8), de la forme $\Omega\left(\frac{z}{u}\right)$; les intégrales (21) et (22) peuvent aussi se réduire à des séries; alors, an lieu de $\Omega(z, u)$, on n' introduit qu' une suite de fonctions $\boldsymbol{\Omega}_{n}(z)$, sans $q \mathbf{u}^{\prime}$ il $\mathbf{y}$ ait d'autre restriction $q \mathbf{u}^{\prime}$ une condition de convergence.

La fonction $Q(z)$ definie par la formule (21) peut se mettre sous la forme (8), comprise au sens large. Mais si, sous cette forme, il subsiste des multiplicateurs négatifs pour certains $\omega\left(\frac{z}{u}\right)$, il est impossible de les faire disparâ. tre. La formule (21) constitue donc une extension de la classe des fonctions de Pólya.

Il nons parait très vraisemblable que les formules (21) et (22) penvent définir des fonctions appartenant à une classe $C_{a}$, mais non à $K_{a}$, et qu'en particulier les sommes finies de la forme (20) où figurent effectivement des termes négatifs ne sont pas réductibles à la forme (9), comprise au sens strict. Mais nous ne l'avons pas démontré. Nous ne savons pas non plus si toutes les fonctions des classes $C_{a}$ peuvent être mises sous la forme (22). Il y a ainsi deux problèmes non résolus qui méritent d'etre étudiés.

V. Nous allons maintenant définir des fonctions de la classe $C$ bien dif. férentes de celles des classes $C_{a}$. Elles ne s'annulent pas nécessairement à l'infini et peuvent être les transformées de FourIER-STIELTJES de fonctions 
discontinues. Mais elles peuvent jouer dans l'étude de l'équation (5) le mème rôle que les fonctions des classes $C_{a}$, parce qu'elles sont nulles sur des ensembles $\mathscr{G}^{c} q$ ui enmprennent des intervalles. Il semble d'ailleurs que n'im. porte quel ensemble $\mathcal{G}^{c}$ fermé, ne contenant pas l'origine, et l'admettant comme centre de symétrie, puisse être considéré comme l'ensemble des zéros de fonctions de la classe $C$. S'il contient des intervalles, ces fonctions peuvent jouer le rôle voulu dans l'étude de léquation (5). Mais nous nous con. tenterons de montrer par des exemples la grande variété des ensembles $\mathfrak{g}^{c}$ possibles.

Partons d'une fonction $q(z) \in C_{a}$, et $\neq 0$ dans $(-a,+a)$. Sa transformée de Fodrier étant absolument continue, elle est de la forme

$$
q(z)=\int_{-\infty}^{+\infty} e^{i z x} f(x) d x \quad[f(x) \geq 0] .
$$

Donnons-nous un nombre $b>a$, et considérons la fonction périodique $P(z)$, de période $2 b$, égale à $q(z)$ dans $(-b,+b)$. Elle est représentable par la série de Fourien

$$
P(z)=\frac{\pi}{b} \sum_{-\infty}^{+\infty} f\left(\frac{\pi h}{b}\right) e^{\frac{\pi i h x}{b}}
$$

dont les coefficients sont $>0$. Elle appartient donc à la classe $C$. Elle est $\neq 0$ dans $(-a,+a)$ et $=0$ dans $[a, 2 b-a]$, et l'onsemble $\mathcal{G}^{c}$ relatif à cette fonction est la réunion des intervalles $[2 k b+a, 2(k+1) b-a]$ (k entier).

Considérons maintenant la somme

$$
S(z)=\Sigma c_{n} P_{n}(z)
$$

et l'expression plus générale

$$
s(z)=\Sigma c_{n} P_{n}(z) \varphi_{n}(z)
$$

où les $P_{n}(z)$ sont des fonctions de la forme (24), $a, b$, et la fonction initiale $q(z)$ variant avec $n$. Les coefficients $c_{n}$ sont positifs, et, s'il s'agit de séries infinies, $\Sigma c_{n} q_{n}$ (o) est supposé fini; les $\varphi_{n}(z)$ sont des fonctions caractéristiques. Alors les séries $S(z)$ et $s(z)$ sont uniformément convergentes.

La somme $S(z)$ est une fonction presque périodique. Si nous supposons les $q_{n}(z)$ réels et $\geq 0$ et les $\varphi_{31}(z)$ réels et $>0, S(z)$ et $s(z)$ sont toujours $\geq 0$, et ne sont nuls que si tous les $P_{n}(z)$ sont nuls. Si $a$ est la borne supérieure des $a_{n}$, si $a^{\prime}$ est la borne inférieure des $2 b_{n}-a_{n}$, et si $a^{\prime}>a$, $S(z)$ et $s(z)$ sont positifs dans $(-a,+a)$, et nuls dans $\left[a, a^{\prime}\right]$, 
et l' ensemble $\mathcal{g}^{c}$ où ces sommes sont nulles ne dépend que des $a_{n}$ et des $b_{n}$. S' il s'agit de sommes finies, cet ensemble a un caractère pres. que périodique, d'où il résulte qu'il comprend une infinité d'intervalles distincts: on peut en effet, à tout $\varepsilon>0$ et $<\frac{a^{\prime}-a}{2}$, faire correspondre des presque périodes $L$ arbitrairement grandes et telles que $\left(a+L+\varepsilon, a^{\prime}\right.$ $+L-\varepsilon) \in \mathcal{O}^{c}$.

Au contraire, s'il s'agit de séries infinies, on peut s'arranger pour que $\mathcal{G}^{c}$ se réduise à l'intervalle $\left[a, a^{\prime}\right]$ et à son symétrique $\left[-a^{\prime},-a\right]$.

Chaque $P(z)$ est en effet $>0$ dans $\left(2 b_{n}-a_{n}, 2 b_{n}+a_{n}\right)$. Pour que $S(z)$ et $s(z)$ soient partout positifs dans $\left(a^{\prime}, \infty\right)$, il suffit de prendre tous les $a_{n}$ égaux à $a, 2 b_{1}=a+a^{\prime}$, et chacun des autres $2 b_{n}-a$ un pen inférieur au plus petit $z>a^{\prime}$ et où les $P_{y}(z)$ d'indices $\vee<n$ sont tous nuls.

On peut naturellement s'arranger pour laisser de côté plusieurs intervalles, et obtenir ainsi des fonctions $S(z)$ et $s(z)$ pour lesquelles $g^{c} \cap(0, \infty)$ com. prenne plusieurs intervalles disjoints $i_{y}$. Mais le moyen le plus simple de montrer qu' on peut identifier $\mathcal{G}^{c} \cap(0, \infty)$ à n'importe quelle réunion finie d'intervalles disjoints situés dans $(o, \infty)$ est de former le produit des fonctions $S_{v}(z)$ formées comme nous venons de le dire, de manière que $S_{v}(z)$ soit nul dans $i_{y}$ et positif dans le reste de la demi-droite $(0, \infty)$. Alors le produit de ces $S_{*}(z)$ est nul dans la réunion des $i_{v}$ et de leurs symétriques et positif partout ailleurs.

On a ainsi, comme nous l'avons dit, une grande variété d'ensembles $\mathcal{G}^{c}$ possibles. A chacun correspond un ensemble très étendu de fonctions $S(z)$ et $s(z)$, puisque, une fois les $a_{n}$ et les $b_{n}$ choisis, on ne change pas $\mathcal{G}^{c}$ en chan. geant les $c_{n}$, les fonctions non négatives $P_{n}(z)$ et les fonctions positives $\varphi_{n}(z)$.

Naturellement, on obtient de nouvelles possibilités en renonçant à ces restrictions sur les signes des fonctions $P_{r}(z)$ et $\varphi_{n}(z)$, et même en introduisant des fonotions imaginaires. Mais alors il peut arriver que $S(z)$ ou $s(z)$ s'annulent sans que tous leurs termes soient nals.

On obtient aussi de nouvelles possibilités en remplaçant les sommes $S(z)$ et $s(z)$ par des intégrales (de LeBesgue ou de Stielnjes). Considérons par exemple une intégrale de la forme

$$
I(z)=\int_{\lambda_{0}}^{\lambda_{1}} P(z, \lambda) f(\lambda) d \lambda \quad[f(\lambda)>0] .
$$

Si la période de $P$ varie avec $\lambda$, le caractère presque périodique de $S(z)$ disparait. Mais il peut exister sur l'axe des $z$ des intervalles où $P(z, \lambda)$ est partout nul, quel que soit $\lambda \in\left(\lambda_{0}, \lambda_{1}\right)$; alors $I(z)$ est aussi nul dans ces intervalles.

VI. Arrivons maintenant au problème essentiel, celui du prolongement dans $\mathcal{G}^{c}$ de la fonction $\varphi(z)$ définie dans $\mathcal{E}$ par la formule (5). Pour qu'il soit 
possible, il faut avant tout que $p(z)$ soit partout nul dans $\mathcal{G}^{c}$, et que les valeurs de $\psi(z)$ données dans $\&$ par la formule (5) définissent une fonction continue dans $\overline{\mathscr{G}}$. Il s'agit alors de la prolonger dans $\overline{\mathcal{G}^{c}}$, en nous plaçant dans le cas, dont nous venons de donner des exemples, où cet ensemble n'est pas vide. Il faudrait d'abord connaitre des conditions nécessaires et suffisantes pour que ce prolongement soit possible. Nous n'arons pas résolu ce problème. Nous allons seulement indiquer des conditions nécessaires, en commençant par les plus triviales.

Nous n'avons pas à nous occuper de la condition que $\varphi(z)$ et $\varphi(-z)$ soient imaginaires conjugués: elle est toujours vérifiée par la fonction $\varphi(z)$ définie dans $\overline{\mathfrak{E}}$ comme nous venons de le dire. Il n'en est pas de meme de la condition $|\varphi(z)| \leq \varphi(0)$, dont on remarque même que, sauf dans le cas où $p(z)=c q(z) e^{e^{\prime} i z}\left(c>0, c^{\prime}\right.$ réel), elle n'est jamais vérifiée à la fois par $\frac{p(z)}{q(z)}$ et par $\frac{q(z)}{p(z)}$. Il faut s'assurer $q u^{\prime}$ elle est vérifiée dans $\overline{\mathscr{E}}$; elle l' est alors dans $\overline{\mathscr{G}}$.

Une autre condition nécessaire simple se déduit de la formule

$$
\cos 2 z x-4 \cos z x+3=2(\cos z x-1)^{2} \geq 0 .
$$

En multipliant par $d F(z)$ et intégrant, il vient

$$
R[p(2 z)-4 \varphi(z)]+3 \varphi(0) \geq 0
$$

et il faut s'assurer qu' elle est vérifiée pour tons les $z \in \overline{\mathfrak{G}}$ el tels que $2 z \in \overline{\mathfrak{G}}$ [ici, il ne suffit pas de le vérifier dans $\mathscr{b}$. Si $(a, 2 a)$ est un des intervalles dont $\overline{\mathcal{G}^{c}}$ est la réanion, il faut vérifier cette condition pour $z=a$, et elle ne résulte pas des vérifications faites quand $z$ et $2 z$ sont des points de $\mathscr{E}$. La mème remarque s'applique aux conditions qui vont suivre].

Plus généralement, considérons une somme ou une intégrale de la forme

$$
\int \cos \lambda z d H(\lambda)
$$

la fonction $H(\lambda)$ étant à variation bornée; $z$ variant de $-\infty a+\infty$, elle a une borne inférieure $m$ et une borne supérieure $M$, et il résulte de la définition (1) de $\varphi(z)$ que

$$
m \varphi(0) \leq R \int \varphi(\lambda z) d H(\lambda) \leq M \varphi(0),
$$


et, s'il existe des $z$ tels que, pour tous les $\lambda$ au voisinage desquels $H(\lambda)$ varie on ait $\lambda z \in \overline{\mathcal{E}}$, c' est une condition imposée aux données ( $\left.{ }^{11}\right)$.

Nous avons ainsi une grande varieté de conditions nécessaires pour la possibilité du prolongement. Il serait peut-ètre intéressant de les réduire au plus petit ensemble de conditions distinctes. Dans le cas réel on peut se demander si l'ensemble de ces conditions ne constitue pas une condition suffisante $\left({ }^{12}\right)$. Dans le cas complexe, on pent appliquer une méthode analogue ì l'étude de l'expression

$$
R \int e^{i \theta} \varphi(\lambda z) d H(\lambda)
$$

qui, an facteur $\varphi(0)$ près, est comprise entre les valeurs extrèmes de

$$
\int \cos (\theta+\lambda z) d H(\lambda)
$$

VII. Plaçons-nous maintenant dans le cas où le prolongement est possible. Comme nous l'avons dit, nous ne connaissons pas de méthode générale pour former tous les prolongements. possibles. Nous allons seulement montrer par des exemples $q u^{\prime}$ il pent arriver que le prolongement soit unique, et $q u^{\prime}$ il peut arriver au contraire $q u^{\prime} i l$ y ait un ensemble très étendu de prolongements possibles. Nous pouvons pour cela sup. poser connu un prolongement $\varphi_{0}(z)$, et, l'ensemble $\&$ étant donné, il s'agit de savoir ce qu' on peut dire de l'ensemble des fonctions $p(z) \in C$ et égales à $\varphi_{0}(x)$ sur 8 .

Un cas où le prolongement est unique est celui où $\varphi_{0}(z)$ est analytique. L'ensemble $\mathfrak{G}$ contient toujours un intervalle $(-a,+a)$. Or on sait qu' une fonction de la classe $C$ analytique dans un tel intervalle est analytique sur tout l'axe réel; l' unicité du prolongement en résulte.

(1i) Pour l'expression $\left.R_{i}^{\Gamma} \varphi(2 z)-4 \varphi(z)\right]$ considerée d'abord, nous n' avons indiqué que la borne inférieuro. La borne supérieure $5 \varphi(0)$ n'est pas meilleure que celle qui résulte de la condition $|\varphi(\lambda z)| S_{\mp(0)}$ appliquée a chaque terme. Si la fonction $H(\lambda)$ est non décroissante, il en sera toujours de mème, et, pour une expression telle que $\varphi(3 z)+\varphi(\varepsilon)$, aucune des deux parties de la formule (30) n'améliore les bornes déduites de $|\varphi(\lambda z)| \leq \varphi(0)$.

(12) Elle serait d'ailleurs pratiquement inapplicable. On remarque que, s'il en est ainsi, cala reste $\nabla$ rai à la limite, quand $\tilde{g}^{\circ}$ est vide, et qu' on aurait théoriquemeni une methode permettant de reconnaitre si une fonction $\varphi(z)$ donnée sur tout l'axe réel appartient à la classe C. Mais la methode rappelée au $n^{\circ} 1$ resterait la seule qui soit pratiquement appli. cable. Cette remarque est à rapprocher de celle faite note (3) à propos des theorèmes de S. Bochner et M. Krein. 
La théorie des fonctions quasi analytiques donne d'autres exemples d'unicité du prolongement. Si la fonction $\varphi(z)$ est indéfiniment dérivable, sa donnée dans $(-a,+a)$ définit tous les moments

$$
E_{n}=\int_{-\infty}^{+\infty} x^{n} d F(x)=(-i)^{n} \varphi_{(n)}(0),
$$

et $\varphi^{(n)}(z)$ est majoré par $\bar{E}_{n}=E_{n}$ si $n$ est pair, et par $\bar{E}_{n}=\sqrt{E_{n-1} E_{n+1}}$ si $n$ est impair. On sait que dans ces conditions, si la série de Denjoy $\Sigma \frac{1}{\sqrt[n]{\bar{E}_{n}}}$ est divergente, $\varphi(z)$ appartient à une classe quasi analytique de, sorte que le prolongement est unique.

Indiquons maintenant au contraire des exemples de prolongements multiples. Un des plus simples est celui des fonctions de Pólra. Si une telle fonction $\varphi(z)$ est donnée dans $(0, a)$ seulement, si $\varphi(a)>0$ et $\varphi^{\prime}(a)<0$, il y a une infinité de manières de prolonger $\varphi(z)$ par des fonctions non croissantes, convexes, et $\geq 0$ à l'infini. Si au contraire $\varphi(z)$ est connu dans $(0, a)$ et dans $(b, \infty)(b>a)$, la condition nécessaire et suffisante pour qu' un tel prolongement soit possible d'une infinité de manières est

$$
\varphi^{\prime}(a)<\frac{\varphi(b)-\varphi(a)}{b-a}<\varphi^{\prime}(b) .
$$

Les courbes représentant les prolongements possibles remplissent alors le triangle compris entre la corde qui joint les extrémités des ares connns et les tangentes en ces deux points.

Il peut d'ailleurs, dans le cas considéré, exister d'autres prolongements que cenx donnés par les fonctions de Pólxa. Ainsi la fonction $2 \omega(2 z)-m(z)$ considérée au no 4 coinncide avec $\omega(3 z)$ dans $\left(0, \frac{1}{3}\right)$ et dans $(1, \infty)$. Si done $g^{c}$ comprend un intervalle $(a, 3 b)(0<a<b)$, et qu' en dehors de cet intervalle on se donne pour $\varphi(z)$ des valeurs contenant des termes de la forme $\omega\left(\frac{3 z}{\lambda}\right)(a \leq \lambda \leq b)$, on des intégralés formées avec la fonction $\omega\left(\frac{3 z}{\lambda}\right)$, on peut indifféremment, pour le prolongement dans $(a, 3 b)$, écrire $\omega\left(\frac{3 z}{\lambda}\right)$, ou

$$
2 \omega\left(\frac{2 z}{\lambda}\right)-\omega\left(\frac{z}{\lambda}\right)
$$

ou plus généralement n'importe quelle moyenne

$$
\mu \omega\left(\frac{3 z}{\lambda}\right)+(1-\mu)\left[2 \omega\left(\frac{2 z}{\lambda}\right)-\omega\left(\frac{z}{\lambda}\right)\right] \quad(0 \leq \mu \leq 1) .
$$


D'ailleurs, en introduisant $2 \omega(2 z)-\omega(z)$, nous n'avons considéré qu'un exemple. Il résulte du $n^{\circ} 4$ que la remarque faite s'étend à beaucoup d'autres combinaisons possibles des fonctions $\theta\left(\frac{z}{\lambda}\right)$. Si notamment $\varphi(z)$ n'est donné que dans un intervalle fini, la variëté des combinaisons possible est immense.

VIII. Au $n^{\circ} 7$, nous n'avons considéré que des fonctions $\varphi(z)$ s'annulant 't l'infini. L'exemple de Khintchine montre que, dans les cas où il y a plusieurs prolongements possibles, il peut exister à la fois, pour prolonger une même fonction donnée dans un intervalle fini, une fonction $s^{\prime}$ annulant à l'infini et une fonction périodique ou presque périodique. Nous allons généraliser ce résultat en montrant que, toutes les fois que les valeurs données dans un intervalle fini $\left(-a^{\prime},+a^{\prime}\right)$ sont celles d' une fonction $\varphi_{0}(z) \in C_{a}$, il $\mathbf{y}$ a une infinité de fonctions périodiques qui prolongent les valeurs données. Cela résulte de la remarque déjà utilisée au $n^{\circ} 5$ : la fonction périodique $P(z)=P(z, b)$, de periode $2 b$, et égale $\varphi_{0}(z)$ dans $(-b,+b)\left[b \geq b_{0}=\operatorname{Max}\right.$ $\left(a, \frac{a+a^{\prime}}{2}\right)$, est une fonction de la classe $C$, qui prolonge les valeurs données. Il en est naturellement de mème de toutes les fonctions de la forme

$$
\varphi(z)=\int_{b_{0}-0}^{\infty} P(z, b) d H(b),
$$

$H(b)$ étant une fonction non décroissante, et qui varie de 0 à 1 dans l'intervalle d'intégration. On a ainsi un ensemble très étendu de fonctions, qui peuvent être périodiques ou presque périodiques, ou au contraire s'annuler, à l'infini, ou encore résulter d'une combinaison de ces deux types.

L'ensemble des solutions est encore plus etendu si on part d' une fonction de Polya $\varphi_{0}(z)$, donnée dans un intervalle $\left(-a^{\prime},+a^{\prime}\right)$ tel que $\varphi_{0}\left(a^{\prime}\right)>0$, $\varphi_{0}^{\prime}\left(a^{\prime}\right)<0$. On a alors une infinité de prolongements, respectant la convexité jusqu'à un point $a$ où la fonction $\varphi(z)$ obtenue est nulle. On peut ensuite, soit supposer que $\varphi(z)$ reste nul, soit introduire les prolongements résultant des remarques finales du $n^{\circ} 7$, ou au contraire ceux de la forme (35). En faisant ensuite varier $a$, en introduisant encore des prolongements convexes de $\varphi_{0}(z)$ qui ne s'annulent qu'à l'infini ou ont une limite positive, et en formant n'importe quelle moyenne pondérée entre toutes ces fonctions, on a une immense variété de prolongements possibles.

La variété est encore plus grande si on part d'un produit, pouvant ctre infini, de fonctions $\varphi_{0}(z)$ du type précédent, puisqne chaque facteur a une infini. tế de prolongements possibles, d'où, pour le produit, un ensemble très étendu de prolongements, qu'on peut étendre à nouveau en faisant des moyennes. Comme nous l'avons déja signalé, les fonctions $\exp \left[\left(-\mid z^{\mid \alpha}\right] \quad(o<\alpha \leq 1)\right.$ 
jouent à ce point de vue un rôle particulier, puisqu' on peut les considérer comme des produits de facteurs $\exp \left[-c \mid z_{i}^{\prime} \alpha\right.$, ou $\exp [-d c|z| x](o<c<1$, $d c>0$ très petit).

Remarquons à ce sujet que, dans une somme (ou un produit) de fonctions de la classe $C$, il suffit $q u^{\prime}$ il $y$ ait un terme (ou un facteur) ayant plusieurs prolongements possibles pour que la somme (ou le produit) ait la mème propriété. Ainsi les fonctions

$$
e^{-|z|-z^{2}}, e^{-|x|}+e^{-z^{2}}
$$

supposées données dans $(-a,+a)$ ont autant de prolongements possibles $q$ ne $e^{-|z|}$, et les fonctions

$$
e^{-|z|-\sqrt{|z|}}, e^{-|z|}+e^{-\sqrt{|z|}}
$$

en ont encore beacoup plus.

\section{Remarque finale.}

Nous sommes très loin de la solution complète des problèmes posés par le prolongement d'une fonction de la classe $C$ donnée dans un intervalle fini. Mais les résultats connns suggérent une remarque: nous ne connaissons aucun exemple, de fonction ' $\varphi(z)$ dérivable à l'origine qui appartienne à une classe $C_{a}$ on qui, sans appartenir à une telle classe, ait plusieurs prolongements possibles. Tous les exemples considérés sont en effet ceux de fonctions vérifiant une inégalité de la forme $(10)\left({ }^{13}\right)$. On peut alors se demander si une telle inégalité ne serait pas une condition, soit nécessaire, soit suffisante, pour que le prolongement en dehors d'un intervalle suffisamment petit $(-a,+a)$ soit indéterminé.

Inversement, nous n'avons démontré l'unicité du prolongement que dans le cas de fonctions indéfiniment dérivables.

Il y a une grande lacune entre ces deux types opposes de fonctions de la classe $C$. Peut-être pourrait-on, pour commencer à la combler, étudier

(13) Cela ne signifie d'ailleure pas nécessairement que la courbe représentant une telle fonction ait a l'origine un point anguleux ou un point de rebroussement: il existe par exemple des fonctions earactéristiques $\varphi(z)$ de la forme $e^{-|z| P(\log |z|)}$ où $P(\log |z|)$ est une fonction périodique oscillant entre deux nombres distincts $a$ et $b$. Alors $\frac{1-\varphi(z)}{|z|}$ oseille, quand $z \rightarrow 0$, entre des bornes téndant vers $a$ et $b$. La courbe représentative d' une telle fonction n'a donc pas a l'origine de demi-tangentes bien définies. Il en est de même pour celle qui représente son produit par $e^{-|z|}$; cette circonstance n'exelut done pas la possibilité du prolongement multiple, impliqué par la présence du facteur $e^{-|\varepsilon|}$. 
le cas des fonctions $e^{-|z|^{\alpha}}(1<\alpha<2)$, qui sont les fonctions caractéristiques de lois stables, ou bien celui des fonctions

$$
\int_{i}^{\infty} \cos z x \frac{d x}{x^{n}}
$$

qui sont $n-1$ fois dérivables. Il s'agirait de savoir s'il y a d'autre fonctions de la classe $C$ qui leur soient égales dans un intervalle $(-a,+a)\left({ }^{14}\right)$.

(14) Depuis que ce travail a été écrit M. M. D. Dugué et E. Lukacs ont attiré mon attention sur un travail de T. Kawata, On the Division of a Probability law, Proc. Imp. Acad. Tokyo», XVI (1940), p. 249-254. Je m'excuse de ne pas l'avoir cité plus haut.

Cet auteur part de la remarque, qui a été aussi utilisée ci-dessus, que le phénomène de Khrntchine n'est possible que si, dans $(0, \infty), p(z)$ est identiquement nul au moins dans un intervalle. A ppliquant alors des résultats d'un travail antérieur, il énonce deux théorèmes (1 et 2) donnant des conditions qui, vérifiées par la fonction de répartition $H(x)$ assocíe à $p(z)$, suffisent pour exclure le phénomène de Khintchine. Il domontre ensuite que ces théorèmes sont les meilleurs possibles. Pour le théorème 2, cela résulte d'un exemple très simple, où intervient un cas particulier de la fonction (24) du présent"travail.

Il me semble d'ailleurs $q u^{\prime}$ il $y$ a une lacune dans l'énoncé de son théorème 2. Les nombres négatifs $a_{n}$ qu'il considère doivent former une suite décroissante, an moins pour $n$ assez grand. La condition $\lim \left|a_{n}-a_{n+1}\right|=\infty$ devient alors $\lim \left(a_{n}-a_{n+1}\right)=\infty$. En effet n'importe quelle suite pour laquelle lim $a_{n}=-\infty$ peut être rangée dans un ordre tel que lim $\left|a_{n}-a_{n+1}\right|=\infty$. Or la condition lim $a_{n}=-\infty$ ne suffit manifestement pas dans l'énoncé du théorème. 